\title{
OTRAS PLATAFORMAS PARA EL ESTUDIO VIRTUAL EN TIEMPO DE EMERGENCIA SANITARIA COVID 19
}

AUTORES: Julissa Rafaela Zambrano Chica ${ }^{1}$

Kirenia Maldonado Zuñiga ${ }^{2}$

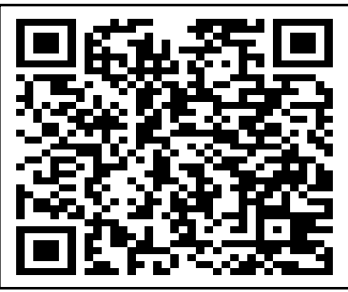

DIRECCIÓN PARA CORRESPONDENCIA: (zambrano-julissa3881@unesum.edu.ec )

Fecha de recepción: 12/02/2021

Fecha de aceptación: 22/08/2021

\section{RESUMEN}

La presente investigación se desarrolló con el objetivo de dar a conocer el uso de las plataformas para el estudio virtual en tiempo de emergencia sanitaria esto atribuye tener sabiduría sobre el funcionamiento que brinda. La importancia de esta investigación es que hoy en día es uno de recursos indispensable para los docente y estudiantes esto permite enriquecer el proceso de aprendizaje-enseñanza, además de brindar y facilitar el intercambio de conocimientos, contenidos de información teniendo más contacto con el estudiante ya se por medio el chat, videollamadas, esto favorece a fomentar el autoaprendizaje como una forma de preparación para que la educación fluya en nuestra vida diaria. Se utilizaron métodos de la investigación científica los cuales se destacaron método histórico-lógico, inducción - deducción y análisis - síntesis y métodos empíricos, los mismo que permitieron el desarrollo de esta investigación, estudiar cada una de las características también se toma como solución al problema que se está viviendo en los actuales momentos. Dentro de los resultados de la investigación se logró realizar un análisis general de las diferentes técnicas que se pueden implementar para el manejo de las herramientas online ayudando así al mejoramiento de los estudiantes en el proceso formativo. Se concluyó que el uso de las plataformas virtuales es importante porque es un medio donde se usa como recurso para la educación esto favorece a la optimización el espacio y mejora la calidad de la información que se está impartiendo.

PALABRAS CLAVE: autoaprendizaje; emergencia sanitaria; fomentar; intercambio.

\footnotetext{
${ }^{1}$ Ingeniera en formación de la carrera Tecnologías de la Información en la Universidad Estatal del Sur de Manabí, Jipijapa - Manabí - Ecuador. Email: zambrano-julissa3881@unesum.edu.ec ORCID: https://orcid.org/0000-00029159-1584

${ }^{2}$ Máster en Ciencias de la Educación, Licenciada en Educación Informática. Docente de la carrera en Ingeniería en Tecnologías de la Información. Universidad Estatal del Sur de Manabí. Jipijapa, Manabí, Ecuador. Email: kirenia.maldonado@unesum.edu.ec ORCID: https://orcid.org/0000-0002-3764-5633
} 


\title{
TITLE OTHER PLATFORMS FOR VIRTUAL STUDY IN TIME OF HEALTH EMERGENCY COVID 19
}

\begin{abstract}
The present research was developed with the objective of publicizing the use of the platforms for virtual study in times of health emergency, this attributes having wisdom about the operation it provides. The importance of this research is that today it is an indispensable resource for teachers and students, this allows enriching the learning-teaching process, in addition to providing and facilitating the exchange of knowledge, information content, having more contact with the student and It is through chat, video calls, this favors promoting self-learning as a way of preparing for education to flow in our daily lives. Scientific research methods were used which highlighted historical-logical method, induction - deduction and analysis - synthesis and empirical methods, the same ones that allowed the development of this research, studying each of the characteristics is also taken as a solution to the problem that is being lived in the current moments. Within the results of the research, a general analysis of the different techniques that can be implemented for the management of online tools was carried out, thus helping the improvement of students in the training process. It was concluded that the use of virtual platforms is important because it is a means where it is used as a resource for education, this favors the optimization of the space and improves the quality of the information that is being imparted.
\end{abstract}

KEYWORDS: exchange; foment; health emergency; Self-learning,

\section{INTRODUCCIÓN}

El uso de plataformas virtuales o herramientas de estudio sirve como una vía de intercomunicación con otros miembros. La evolución de las plataformas educativas se muestra muy ligada al desarrollo de la sociedad de la información y del conocimiento, en concreto, al de los sistemas educativos, que tienden, progresivamente, a adaptarse a las necesidades reales del mundo laboral. En este marco de innovación y cambio, la opción de generar entornos virtuales de aprendizaje basados en las tecnologías de la información y la comunicación (TICS), supone responder de forma integral a los requerimientos de las nuevas necesidades del entorno educativo. (Becerro)

Es dentro de este contexto de innovación donde surgen las plataformas educativas virtuales como es Google Classrooms, esta es una herramienta que ayuda acelerar los procesos de comunicación entre los docentes y los estudiantes al momento de realizar la tares es partica para el proceso de enseñanza-aprendizaje. (mirplayschool, 2020)

98 UNESUM-Ciencias. Publicación cuatrimestral. Vol. 5, Año 2021, No. 5 (Septiembre-Diciembre) 
Chamilo es una herramienta la cual permite a los docentes construir cursos online esto dispone de una serie de metodologías para el funcionamiento del desarrollo de las tareas. Está construido bajo la óptica de construcción del conocimiento basado en el diálogo entre los participantes y el constructivismo social. Sakai destaca por tener la opción de videoconferencia, que ha tenido buena aceptación. Además, divide la función de sus herramientas según el objetivo del aprendizaje; son generales de colaboración, administrativas y de portafolios. Edmodo pone como punto clave la comunicación y el profesor puede crear las aulas virtuales. (mirplayschool, 2020)

Correo electrónico es otro medio de que el docente puede asignar tareas o trabajos, así como recibirlos, revisarlos y proporcionar retroalimentación directa a los estudiantes. Chats es una herramienta de comunicación sincrónica en el que se utilizan mensajes escritos permite interactuar en tiempo real, por el intercambio de ideas y la colaboración. Foros virtuales es un medio de interacción asincrónica. (Hernández, 2010)

El objetivo de esta investigación es dar a conocer el uso de las plataformas virtuales en tiempos de emergencia sanitaria Covid 19. El impacto es que la educación virtual a dado un giro en el uso de las plataformas como una herramienta para facilitar el proceso educativo de esta manera que facilita la trasmisión de conocimientos entre los docentes y los estudiantes con esto se pretenden hacer un reemplazo entre las aulas tradicionales para formatos virtuales.

\section{DESARROLLO}

Una plataforma educativa virtual es un esquema que abarca otros tipos de herramientas destinadas a fines docentes. Su primordial función es proporcionar la creación de entornos virtuales para compartir cualquier tipo de alineaciones a través de internet sin necesidad de tener preparaciones de programación.

En realidad, son programas que crear tareas como: la creación de contenidos y diferentes actividades entre de un curso online, trámites de matrículas de los estudiantes, obtener una búsqueda del trabajo durante la carrera, solucionar dudas y establecer espacios de información interactiva, valorar el avance de los estudiantes, etc.

Se utiliza para organizar de forma integral alineaciones a distancia o como un mejoramiento de la enseñanza presencial. Permite establecer áreas de discusión y trabajo para los grupos de indagación, o para efectuar comunidades virtuales y tramas de enseñanza en torno a los temas de interés común. (Prados, 2017) 
Las plataformas digitales, son lugares en web que acceden a la realización de varias aplicaciones o programas en un propio lugar para satisfacer diferentes necesidades. Estas cuentan con diferentes funciones que ayudan a los beneficiarios a solucionar diferentes tipos de dificultades de forma computarizada, utilizando bajo recursos.

El primordial objetivo que desempeñan las plataformas digitales es proporcionar la realización de tareas a través de programas o aplicación en un propio lugar del internet de acuerdo con la necesidad de los usuarios. (Giraldo, 2020)

\section{Google Classroom}

Google Classroom es la herramienta de Google para la formación. Es una plataforma que consiente gestionar lo que sucede en la clase de forma online, de modo colaborativa. Inició el año 2014, y su uso ha tenido un crecimiento exponencial entre los educadores de diferentes partes del mundo. Las diversas funcionalidades de Google que brinda al entorno de la alineación, están relacionadas a una cuenta GMAIL y ofrece la posibilidad de formar documentaciones; compartir información en diferentes formas (vídeos, hojas de cálculo, presentaciones y más), agenda reuniones y llevarlas a cabo de manera online, entre otros varios propósitos.

Esta herramienta admite tramitar el aprendizaje a distancia o mixto (semipresencial), en los grupos educativos, logra aprobar a partir de diferentes unidades mejorando el camino sin importar el lugar ni la hora.

\section{Ventajas de Google Classroom}

A modo que hemos definido, las ventajas de esta plataforma forman podemos decir que facilitan y mejoran el trabajo del docente.

- Logran establecer clases interactivas con docentes de otros cursos y otras materias.

- Obtienen el control de los trabajos de los alumnos.

- Facilita la evaluación de exámenes y trabajos de los alumnos.

- Incremento la información en la agrupación educativa. (Innedu, 2018)

\section{CHAMILO}

Chamilo LMS es una herramienta LMS OPENSOURCE bajo la licencia GNU/GLP v3 (Licencia Pública General), se instalar, cambiar y crear elementos que admitan poder ajustar esta plataforma a necesidades concretas de proyectos de elearning. Hay que recalcar que esta herramienta fue desarrollada por la asociación Chamilo en base al código de Dokeos. Chamilo se ha transformado en una plataforma de enseñanza-aprendizaje en línea muy popular en los en los actuales momentos entre el sector educativo, también es gratis, permite la modificación y distribución de contenidos, de carácter sencilla y bajo una orden de ordenación suficiente completo.

\section{Las principales funcionalidades de Chamilo son:}

- Creación de cursos: logras establecer cursos y añadir el material que requieras para realizar los trabajos. 
- Gestión de beneficiarios: logras ejecutar sus inscripciones y asignación de docentes y estudiantes a tus cursos.

- Valoración y capacidades

\section{Ventajas y desventajas \\ Ventajas \\ - Se logra personificar.}

- Tiene materiales de búsqueda y gestión.

- Comunicaciones de programas y tareas.

- Trabajos colaborativos en grupo.

- Admite paquetes SCORM.

- Admite una gran gestión documentos.

- Videoconferencia.

\section{Desventajas}

- Precisas instalarla en un servidor para poder ofrecer cursos por espacio virtual.

- No peen de un marketplace para la comercialización de los cursos.

- Solicita ser actualizada continuamente.

- Es dificultoso de manipular

- Si bien es una plataforma muy perfecciona y con una interfaz abierta, la práctica de beneficiario es terrible. (Linares, 2020)

\section{SAKAI}

Es un ambiente modular de código fuente abierta (Software Libre), cuyo objetivo es formar varias funcionalidades del E-learning en un portal académico. Está garantizado por las mejores instituciones del mundo y preservada por un extenso grupo de técnicos. A la forma Open Source o código abierto consta de varias ampliaciones e incrementa, cubriendo de esta representación de los acontecimientos necesidades que surgen en el mundo del e-learning.

\section{Características Principales:}

Al igual que el resto de estas herramientas estudiadas, Sakai domina los instrumentos primordiales de toda plataforma de aprendizaje virtual. Sakai forma un especial hincapié en los mecanismos destinadas a la creación de materiales por parte del estudiante y a la indagación de sus trabajos que da el docente al estudiante. En exclusiva las implementaciones llamadas de portafolio alineadas al estudiante. (plataformaasvirtuales, 2016)

\section{EDMODO}

Edmodo es una red formativa que obtiene como finalidad facilitar a los maestros como instrumento que ayude a notificar con sus estudiantes. A través de la herramienta los docentes alcanzan compartir contenidos, argumentos, vídeos y trabajos con sus estudiantes. Los 
instrumentos online para ayudar a los maestros a dirigir sus clases han desarrollado de conocimientos se conocen como Sistemas de Gestión de Aprendizaje (LMSs).

Utilizando Edmodo, los estudiantes y los docentes realizan a lograr a vincular compartiendo opiniones, dificultades y consejos útiles. Un profesor consigue fijar y examinar la tarea encargada Edmodo, se realizan apoyo de cualquiera la clase. También se presenta de un ambiente seguro.

Formando un ambiente colaborativo, pero además positivo en donde el desarrollo de los estudiantes consigue ser guiado en todo tiempo que sea necesario impidiendo conductas no adecuados. En la realidad lograríamos limitar a Edmodo como una red social privada, por lo que ninguno externo al ambiente formativo o la clase manejada por un docente, o por un establecimiento, posee acceso ni obtiene a conectar u observar esa red.

\section{Características de Edmodo}

Edmodo se ocupa directamente de las preocupaciones de los establecimientos y de los docentes sobre las redes sociales para los estudiantes del consecutivo modo:

- Cada grupo de clase de modo lo dirige y controla por el docente.

- Los estudiantes requieren un clave de acceso para unirse a la clase. Si un estudiante difunde la contraseña fuera de la clase, el maestro puede cambiarlo.

- Los estudiantes sólo consiguen notificar con toda la clase o con el profesor.

- La dirección de los papás a los puestos de sus hijos y a los docentes es una cualidad opcional. (Toribio, 2019)

\section{CORREO ELECTRÓNICO}

El correo electrónico, también conocido como e-mail, es un servicio de red que admite disponer y recibir envíos de mensajes con varios receptores, ubicados en cualquier parte del mundo. Este servicio se requiere en cualquiera de las trasmisiones de correo electrónico que brinda la red. En un envío de correo electrónico, también de un argumento escrito, logra contener archivos tales como documentaciones, imágenes, canción, registros de video, etc. Mejoran el uso, rapidez y el bajo costo de la entrega la culés se realiza a la mayoría de los establecimientos y particulares obtengan el correo electrónico como primordial intermediario de comunicación. (Pérez, 2020)

\section{CHATS}

El término chat se refiere a un tipo de información digital que se proporciona a través de la red de web entre dos o más usuarios. La comunicación por chat se logra transportar por envíos de texto, videollamadas. Los chats son pláticas que se producen al momento gracias al uso de un software acoplado a una red de Internet y se distinguen por forma chats públicos, conjuntos de diálogo en los cuales logra notificar cualquier individuo o, chats privados, que solo obtienen notificar beneficiarios delegados.

El objetivo fundamental de los chats es alcanzar que los individuos se informen e intercambien investigación al momento sin importar adonde se localicen, disminuyendo de este modo las barreras del recorrido y el tiempo de modo mucho más económica. Los chats fueron establecidos 
como un canal de información y cambio de investigación, sobre todo en la actualidad, en donde los usuarios, se enlazan continuamente a las redes para estar al tanto acerca de lo que sucede en el mundo y para lograr o compartir investigación. (Significados, 2017)

\section{MATERIALES Y MÉTODOS}

Se utilizaron los métodos de investigación científica, predominando los métodos de inducción deducción y análisis - síntesis, que contribuyeron en el desarrollo y así mismos en poder dar a conocer las plataformas de estudio virtual.

\section{Métodos teóricos}

\section{Método histórico - lógico}

Se utilizó este método de investigación para analizar los antecedentes de las plataformas de estudio virtuales que aporta para la investigación.

\section{Método análisis - síntesis}

Se utilizó para poder proporcionar y conocer cada una de las actividades que plante la investigación la cual son de gran importancia.

\section{Método inducción - deducción}

Este método contribuyó en la investigación para poder realizar los procedimientos que son esenciales para la investigación también para llegar al tema central que es otras plataformas para el estudio virtual en tiempo de emergencia sanitaria covid 19.

\section{Métodos empíricos}

Estos se emplean fundamentalmente de acumulación de información empírica en esta investigación se hizo énfasis en los siguientes métodos:

\section{Observación}

Esta técnica de investigación se aplicó para observar y partir de estas sacar un diagnóstico sobre el uso de las diferentes plataformas virtuales educativas.

\section{Revisión bibliográfica}

Con este método se pudo complementar la aplicación del método histórico - lógico en la investigación. 
Julissa Rafaela Zambrano Chi, Kirenia Maldonado Zuñiga

\section{RESULTADOS Y DISCUSIÓN}

Se obtuvo como resultado que las plataformas virtuales el último tiempo han ido incrementando del porcentaje a la hora de la utilización para esto se logró que los estudiantes tengan conocimientos del ¿Qué es? y el ¿Para qué es? los beneficios de lo que es las plataformas virtuales sus ventajas, desventajas y la importancia con la que cuenta como parte fundamental de la por medio de esto pueden darse soluciones sencillas fácilmente que puedan ser aplicadas en los estudiantes sino a quien lo requiera para fortalecer el conocimiento. Dado esto se obtuvo que los estudiantes cuenten con los conocimientos adecuados y suficientes para saber los que son las plataformas de estudio virtual.

Esta investigación surge de la necesidad de motivar a los docentes y estudiantes que sigan investigando sobre las plataformas de estudio virtuales e integren las TIC en sus aulas de clase, implementando ambientes de aprendizaje fortalecidos por estas tecnologías, para favorecer y mejorar las relaciones entre educadores y estudiantes.

Las nuevas tecnologías pueden suministrar medios para la mejora de los procesos de enseñanzaaprendizaje. Para la gestión de los entornos educativos en general, pueden facilitar la colaboración entre las familias, los centros educativos, el mundo laboral y los medios de comunicación, pueden proporcionar medios para hacer llegar en todo momento y en cualquier lugar la formación que la sociedad exija a cada ciudadano, y también contribuir a superar a la sociedad.

\section{CONCLUSIONES}

Se puede afirmar que las plataformas de estudio virtual están completamente establecidas al soporte de la perspectiva tecnológica como didáctica para los estudiantes y docentes para la contrición de conocimiento y aprendizaje.

Las herramientas online en la actualidad han influenciado decisivamente para la trasformación tradición en los hábitos de aprendizaje para los estudiantes, porque son equipos que se utilizan todos los días, lo importante es aprender usar el diferente elemento dinámico básico para impartir las clases mediante la tecnología que ayuda hacer los procesos más fáciles.

Se encontró que los entrones virtuales brindan medios adecuados para la enseñanza- aprendizaje al momento que los docentes impartes su conocimiento a los estudiantes.

\section{REFERENCIAS BIBLIOGRÁFICAS}

Becerro, S. D. (s.f.). feandalucia. Obtenido de feandalucia: https://www.feandalucia.ccoo.es/docu/p5sd4920.pdf Giraldo, V. (22 de 07 de 2020). Obtenido de https://rockcontent.com/es/blog/plataformas-digitales/

Hernández, J. A. (13 de 08 de 2010). Docentes al Día. Obtenido de Docentes al Día: https://docentesaldia.com/2020/03/15/8-herramientas-e-ideas-para-implementar-la-ensenanza-a-distancia-entu-escuela/

Innedu, B. (17 de 09 de 2018). Obtenido de https://www.innedu.es/bootcamps/google-classroom-que-es-y-para-quesirve/

Linares, J. C. (30 de 10 de 2020). Obtenido de https://bit4learn.com/es/lms/chamilo/

104 UNESUM-Ciencias. Publicación cuatrimestral. Vol. 5, Año 2021, No. 5 (Septiembre-Diciembre) 
mirplayschool. (26 de 03 de 2020). Obtenido de mirplayschool: https://www.mirplayschool.com/5-herramientaspara-la-educacion-virtual/

plataformaasvirtuales. (02 de 11 de 2016). Obtenido de plataformaasvirtuales: http://plataformaasvirtuales.blogspot.com/2016/11/plataforma-sakai.html

Prados, E. (23 de 11 de 2017). Obtenido de https://www.aula1.com/plataformaseducativas/\#: :text=Una\%20plataforma\%20educativa\%20virtual\%20es,de\%20tener\%20conocimientos\%20d e\%20programaci\%C3\%B3n.

Pérez, M. (18 de 11 de 2020). Obtenido de https://conceptodefinicion.de/correo-electronico/ Significados. (13 de 06 de 2017). Obtenido de Significados: https:/www.significados.com/chat/

Toribio, C. V. (28 de 08 de 2019). Recuperado el La Educación en la era Digital de La Educación en la era Digital, de https://ayto-torrijos.com/educacion/que-es-edmodo/ 
106 UNESUM-Ciencias. Publicación cuatrimestral. Vol. 5, Año 2021, No. 5 (Septiembre-Diciembre) 